\title{
Local and Semi-Global Feature-Correlative Techniques for Face Recognition
}

\author{
Asaad Noori Hashim \\ Dept. of Computer Science \\ University of Babylon \\ P.O.Box 4, Babylon, \\ Iraq
}

\author{
Zahir M. Hussain \\ Dept. of Computer Science \\ University of Kufa \\ P.O.Box 21, Kufa, Najaf, Iraq \\ Adjunct Professor, ECU, Australia
}

\begin{abstract}
Face recognition is an interesting field of computer vision with many commercial and scientific applications. It is considered as a very hot topic and challenging problem at the moment. Many methods and techniques have been proposed and applied for this purpose, such as neural networks, PCA, Gabor filtering, etc. Each approach has its weaknesses as well as its points of strength. This paper introduces a highly efficient method for the recognition of human faces in digital images using a new feature extraction method that combines the global and local information in different views (poses) of facial images. Feature extraction techniques are applied on the images (faces) based on Zernike moments and structural similarity measure (SSIM) with local and semi-global blocks. Preprocessing is carried out whenever needed, and numbers of measurements are derived. More specifically, instead of the usual approach for applying statistics or structural methods only, the proposed methodology integrates higher-order representation patterns extracted by Zernike moments with a modified version of SSIM (M-SSIM). Individual measurements and metrics resulted from mixed SSIM and Zernike-based approaches give a powerful recognition tool with great results. Experiments reveal that correlative Zernike vectors give a better discriminant compared with using $2 \mathrm{D}$ correlation of the image itself. The recognition rate using ORL Database of Faces reaches 98.75\%, while using FEI (Brazilian) Face Database we got $96.57 \%$. The proposed approach is robust against rotation and noise.
\end{abstract}

Keywords-Zernike Moments; Face Recognition; Structural Similarity

\section{INTRODUCTION}

Face recognition has become one of the most successful applications of image analysis and computer vision. Face recognition software has been incorporated in a wide variety of biometrics-based security systems for the purposes of identification, authentication and video surveillance. Face recognition includes three stages. The first stage is detecting the location of the face, which is a difficult task for the position, orientation, and scaling of the face are unknown in an arbitrary image. The second stage involves extraction of the pertinent features of the localized facial image obtained in the first stage. Finally, the third stage requires classification of facial images based on the derived feature vector obtained in the previous stage.

Unlike humans who have an outstanding capability of recognizing different patterns and faces in varying conditions, machines are still dependent on ideal face images; their performance suffers when there are variations in illumination, background, pose angle, obstacles, etc. Therefore, the problem of automatic face recognition is a very complex and challenging task [1]. Conventionally, face recognition methods are classified in two categories. The first one is based on extracting structural facial features that are local features of face images, for example, the shapes of eyes, nose and mouth. The structure- based approaches deal with local information instead of global information. The second category is based on statistical approaches, wherein features are extracted from the whole image and thus use global information instead of local information. Since the global data of an image are used to determine the feature elements, data that are irrelevant to facial portion such as hair, glasses, shoulders and background may result in the creation of erroneous feature vectors that can affect the recognition results [2].

Statistical approaches for feature extraction based on moment invariants have been utilized for classification and recognition applications because of their invariance properties. An image feature is considered invariant if it remains neutral to changes in size (scale), position (translation), orientation (rotation), or/and reflection in an image. The most popular appearance - based face recognition algorithms are: Principal Component Analysis (PCA), Independent Component Analysis (ICA), and Linear Discriminant Analysis (LDA). PCA finds a set of the most representative projection vectors such that the projected samples retain most of the information about original samples. ICA captures both second and higher-order statistics and projects the input data onto the basis vectors that are as statistically independent as possible. LDA uses the class information and finds a set of vectors that maximize the between-class scatter while minimizing the within-class scatter [3].

Pan and Bolouri (1999) used the discrete cosine transform to reduce image information redundancy, because only a subset of the transform coefficients are necessary to preserve the most important facial features such as hair, eyes and mouth. The researchers state that when DCT coefficients are fed into back propagation neural network for classification, high recognition rate can be achieved by using a very small proportion of transform coefficients [4]. Hafed et al. (2001) introduced an accurate and robust face recognition system. This system exploits the feature extraction capabilities of the discrete cosine transform (DCT) and invokes certain 
normalization techniques that increase robustness to variations in facial geometry and illumination [5]. Hazim et al. (2005) proposed a local appearance- based face recognition algorithm, where local information is extracted using blockbased discrete cosine transformation; and obtained local features are combined both at the feature level and the decision level [6].

In [7], a novel algorithm was proposed by Osslan et al. (2009) to solve the problem of automatic face recognition is presented; where the novelty of the algorithm is the ability to combine the computer vision tasks with Particle Swarm Optimization (PSO) to improve the execution time and to obtain better recognition results. The crucial stage of a typical system of face recognition has been improved by using a fitness function to measure the similarity of the input face compared with a database of faces. Sharma et al. (2010) introduced simple but efficient novel H-eigenface (Hybrideigenface) method for pose- invariant face recognition, ranging from frontal to profile view. The proposed method is based on the fact that face samples of same person under different poses are similar in terms of the combination patterns of facial features [9]. Zhang and $\mathrm{Li}$ (2010) proposed discriminative KSVD (D-KSVD) based on extending the K-SVD algorithm by incorporating the classification error into the objective function[10].

Lone et al. (2011) used a multi-algorithmic approach, where they developed a face recognition systems based on one combination of four individual techniques, namely, Principal Component Analysis (PCA), Discrete Cosine Transform (DCT), Template Matching using Correlation (Corr) and Partitioned Iterative Function System (PIFS). Researchers fuse the scores of all of these four techniques in a single face recognition system [11].

Invariants, especially Zernike moments, also adopted by researchers and became more attractive due to its robustness against rotation and noise. Shi et al. (2012) proposed a feature extraction method based on pseudo-Zernike moment followed by LDA for dimensionality reduction [12].

Singh et al. (2012) proposed a modified PCA algorithm by using some components of the LDA algorithm for face recognition. The algorithm is based on the measure of the principal components of the faces, then to find the shortest distance between them [13]. On the other hand, many researchers utilize local features embedded within the human face. Manchula and Arumugam (2013) presented a featurebased multimodality face recognition system to recognize the human individuals in an environment of known faces using features like shape of the eyes, nose and jaw [14].

We outline this paper as follows: the next section describes the proposed system which consists of a number of steps: Image Pre-Processing, Image Dividing and Feature Extraction (such as Zernike Moments), Modified Structural Similarity Index Measurements (M-SSIM), Features Selection, Measurement Performance (that illustrate a number of main and derived measurements for face recognition), and the final step of proposed system, which is Classification. Section 3 explains the standard data sets which used for face recognition.
The remaining sections are Contributions, Results, Conclusions, and Discussions.

\section{LOCAL AND SEMI-GLOBAL FEATURE EXTRACTION}

Different emotions and occlusions are represented by facial features with more emphasis on specific areas of the face than other areas (center of the face); also, changing lighting conditions (lighting direction and illumination condition) are considered. In this paper, a novel weighted patch moment array face representation and recognition approach is introduced. The overall framework of the algorithm is illustrated in the following steps. There are four main steps in the proposed algorithm:
1) Pre-processing operations,
2) Image partitioning and feature extraction,
3) Measurements performance,
4) Classification.

\section{A. Image Pre-Processing}

The following pre-processing operations are needed before applying the proposed algorithm:

1) Modifying image scales: All images must be square and have even dimensions.

2) Specifying the order and repetition of Zernike to get a set of polynomials, using the following algorithm.

3) Preparing window parameters for SSIM.

4) Face detection: face detection is a necessary preprocessing stage. However, it is a research issue by itself, with major difficulties and challenges. Therefore, we propose our approach based on standard datasets, which provide suitable (pre-processed) images for recognition without the need to face detection stage.

Algorithm (1): Zernike Order-Repetition Set

Initialization: $\quad \mathrm{m}=[\mathrm{]}, \mathrm{n}=[\mathrm{]}$

Input: $\quad$ Po $\quad$ //Minimum Order

Output: Zernike Order-Repetition Set

Zernike Set:

$$
\begin{aligned}
& \text { for } h=P o \text { to } P \\
& \text { for } \mathrm{f}=0 \text { to } \mathrm{P} \\
& \text { if }(\mathrm{f}<=\mathrm{h} \text { and } \bmod (\mathrm{f}-\mathrm{h}, 2)=0) \\
& \mathrm{n}=[\mathrm{n} \mathrm{h}] \\
& \mathrm{m}=[\mathrm{m} \mathrm{f}] \\
& \text { end if } \\
& \text { end loop } \mathrm{f}
\end{aligned}
$$

end loop $\mathrm{h}$

\section{B. Image Dividing and Feature Extraction}

Choosing an efficient feature extraction method is the most important factor to achieve a high recognition rate in face recognition. In the proposed algorithm, a human face image is divided into a set of equal-sized blocks in an overlapping manner. In this work, the dimension of each face image training image or test (reference) is $\mathrm{N}^{*} \mathrm{~N}=92 * 92$ pixels, the following algorithm is used to divide each image in to overlapping windows. 
Algorithm (2): Dividing images y,x into overlapping blocks Initialization:

$\mathrm{wg}=[6242], \mathrm{sg}=[1525] / /$ Global/Local windows

$\mathrm{yw}=[\mathrm{]}, \mathrm{xw}=[\mathrm{]} / / \mathrm{yw}$ sub-block of test face

//xw sub-block of training image

Input: $y$ is reference (test) image with size $\mathrm{N} * \mathrm{~N}$

$x$ is training image with size $\mathrm{N} * \mathrm{~N}$

Output: $\mathrm{y}$ and $\mathrm{x}$ are divided into sub-blocks $\{\mathrm{yw}, \mathrm{xw}\}$

Step One: Compute the length of wg: $\mathrm{Lg}=$ lenght(wg) ;

Step Two: Partition Images

For $\mathrm{g}=1$ to $\mathrm{Lg}$

$\mathrm{w}=\mathrm{wg}(\mathrm{g}) ; \mathrm{s}=\mathrm{sg}(\mathrm{g}) ; \mathrm{k}=$ round $(\mathrm{N}-\mathrm{w}) / \mathrm{s}$

for $\mathrm{s} 1=0$ to $\mathrm{k} ; \mathrm{s} 2=\mathrm{s} 1 * \mathrm{~s}$

for $\mathrm{w} 1=0$ to $\mathrm{k} ; \mathrm{w} 2=\mathrm{w} 1 * \mathrm{~s}$

$\mathrm{yw}=\mathrm{y}(\mathrm{s} 2+1: \mathrm{s} 2+\mathrm{w}, \mathrm{w} 2+1: \mathrm{w} 2+\mathrm{w}) ;$

$\mathrm{xw}=\mathrm{x}(\mathrm{s} 2+1: \mathrm{s} 2+\mathrm{W}, \mathrm{w} 2+1: \mathrm{w} 2+\mathrm{W}) ;$

end loop w1

end loop s1

end loop g

\section{1) Zernike Moments}

Mmoments compute a numeric quantity at some distance from a reference point or axis. While Zernike polynomials are defined as a set of orthogonal polynomials defined on the unit disk, Zernike moment is the projection of the image function onto these orthogonal basis functions. Zernike moments have been proven to be more robust in the presence of noise. Since their moment functions are defined using polar coordinate representation of the image space, Zernike moments are commonly used in recognition tasks requiring rotation invariance. Zernike moments are a good feature representation and provide more information about facial image and reduce the dimension of the feature vector leading to improved results. Implementation of Zernike moments is detailed in [15]; while some formulas were corrected by Sun-Kyoo Hwang. The kernel of Zernike moments is a set of orthogonal Zernike polynomials defined over the polar coordinate space inside the unit circle. Zernike moments of order $p$ with repetition $q$ of an image with intensity $f(r, \theta)$ are defined as follows [16]:

$Z_{p q}=\frac{p+1}{\pi} \int_{0}^{2 \pi} \int_{0}^{1} R_{p q}^{*}(r) e^{j q \theta} f(r, \theta) r d r d \theta, \quad|r| \leq 1$

where the radial polynomial $R_{p q}(r)$ is given as follows:

$R_{p q}(r)=\sum_{k=0}^{\frac{p-|q|}{2}}(-1)^{k} \frac{(p-k) !}{k !\left(\frac{p+|q|}{2}-k\right) !\left(\frac{p+|q|}{2}+1-k\right) !}$

with $0 \leq|q| \leq p$ and $p-|q| \mid$ is even.

Zernike moments utilize polar coordinates $(r, \theta)$ inside the unit circle $|r| \leq 1$. To approximate and compute them in discrete form we perform a linear transformation of the image Cartesian coordinates ( $i, j$ ) from the inside of the square $i, j=0,1, \ldots, N-1$ to the inside of the unit circle $|r| \leq 1$ to get the discrete form:

$$
Z_{p q}=\lambda_{z}(p, N) \sum_{i=0}^{N-1} \sum_{i=0}^{N-1} R_{p q}\left(r_{i j}\right) e^{-j q \theta_{i j}} f(i, j)
$$

where

$$
\left.\begin{array}{ll}
r_{i j}=\sqrt{x_{i}^{2}+y_{j}^{2}} ; & \theta_{i j}=\tan ^{-1}\left\{\frac{y_{j}}{x_{i}}\right\} \\
x_{i}=\frac{2 i}{N-1}-1 ; & y_{j}=\frac{2 j}{N-1}-1 \\
\lambda_{p}(p, N)=\frac{p+1}{N-1} &
\end{array}\right\}
$$

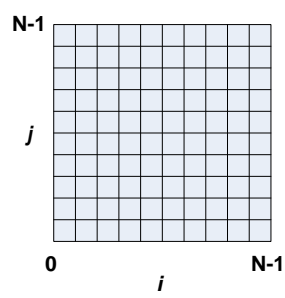

Fig. 1. Two Coordinate normalization schemes for radial and Zernike moments: (a) Discrete image coordinate space of size $(\mathrm{N} * \mathrm{~N})$; (b) Coordinate normalization using map $(0, \mathrm{~N}-1) \rightarrow(-1,+1)$.

The above coordinate's transformation is shown in Figure 1. The figure illustrates that, the algorithm will focus on the center of an image, which includes the human face directly, this will increase the accuracy of recognition with small redundancy. The radial moments used here are complex in nature. Since their magnitude is invariant to rotation, so we are utilizing just their magnitude as a feature vector with several orders. Zernike moment is used as a feature extractor the value of order $\mathrm{p}$, repetition $\mathrm{q}$, which is varied to achieve the best classification performance.

2) Modified Structural Similarity Index Measurements $(M-S S I M)$

An objective image quality measure can have a significant role in image processing and its applications, where it can be used to monitor and adjust image quality. Also, a quality measure can be used to optimize algorithms and parameter settings of image processing systems. Machine evaluation of image and video quality is important for many image processing systems, for example, systems used for compression, restoration, enhancement, etc. The goal of quality assessment is to find robust techniques for objective evaluation of image quality in accord with subjective human assessment. Wang et al. (2004) [17] proposed a promising technique (SSIM) for distance covariance to measuring the structural similarity based on number of statistical measurements such as mean, standard deviation and they produced a new relation among these standards as $\mathrm{n}$ the following formula:

$$
p(x, y)=\frac{\left(2 \mu_{x} \mu_{y}+C_{1}\right)\left(\sigma_{x y}+C_{2}\right)}{\left(\mu_{x}^{2}+\mu_{y}^{2}+C_{1}\right)\left(\sigma_{x}^{2}+\sigma_{y}^{2}+C_{2}\right)}
$$

where, $\rho(x, y)$ is the SSIM measure between two images $x$ and $y, \mu x$ and $\sigma_{x}^{2}$ are the statistical mean and variance of pixels in image $\mathrm{x}\left(\mu \mathrm{y}, \sigma_{\mathrm{y}}^{2}\right.$ are defined similarly), $\sigma_{\mathrm{xy}}$ is the statistical variance between pixels in images $\mathrm{x}$ and $\mathrm{y}$, while the constants $\mathrm{C} 1$ and $\mathrm{C} 2$ are defined as $\mathrm{C} 1=(\mathrm{K} 1 \mathrm{~L}) 2$ and $\mathrm{C} 2=(\mathrm{K} 2 \mathrm{~L}) 2$, 
with $\mathrm{K} 1$ and $\mathrm{K} 2$ are small constants and $\mathrm{L}=255$ (maximum pixel value). This approach gives a high level of similarity for noise free condition while it goes to zero when noise increase, in other words, it gives a similarity with two different images due to it dependent only the statistics features of images which may have some correlations. SSIM can't reveal all image structural properties, so we need to more specific measurements that are image-dependent. Pure SSIM gives a good results, but if it combined with Edge detection filters such as Canny, it will produce excellent results specially when the images are different from each other (in this case well return zero value for similarity) [18].

\section{NeW MEASURES: A FEATURE-CORRELATIVE APPROACH}

In previous works, we used either local or global features for recognition successfully[19-21]. In this work, global features proved ineffective, and we use mixed local and semiglobal features. Seven measurements are investigated: First three are tested individually, while others are combined in some ways. Zernike moment is applied on equal size of overlapping blocks in a local manner (small window) and semi-global (large window). In general, the main measurements are Zernike correlation, Zernike errors, and SSIM. Each one of these measurements must be converted into one dimension before using the other measurements. In each measurement we tried to find first and second maximum values, where the first represent the required person with high probability while the second represent the person with probability less than the first. To satisfy above goals, many normalizations operations as well as dimensions reductions are applied. The measurements are explained later.

\section{A. Zernike Correlation Measure}

Correlation measures the linear relationship between any two variables, and if these variables are independent, the correlation will be approximately zero. In addition, correlation matrices give an overview of the pattern of relationships between variables.

With the proposed algorithm, Pearson correlation coefficients are computed to find the autocorrelation of Zernike moments of (windowed) reference image and cross correlation between the Zernike moments of (windowed) reference image and moments of (windowed) poses of database. Then the minimum distance between the two correlations over all possible windows will indicate a measure of similarity (recognition). The following formulas are used, with $\mathrm{y}_{\mathrm{w}}$ indicating the $w$-th window of the reference image and $x_{w}$ indicating the w-th window of the test pose:

$e_{c}(x, y)=\min _{w}\left[\sum_{i=1}^{2 L_{z}-1} \frac{\left\{R_{x_{w} y_{w}}(i)-R_{y_{w}}(i)\right\}^{2}}{M^{2}}\right]$

where

$R_{x_{w} y_{w}}=\operatorname{xcorr}\left\{\left|Z_{P_{o} P}\left(x_{w}\right)\right|,\left|Z_{P_{o} P}\left(y_{w}\right)\right|\right\}$,

$R_{y_{w}}=\operatorname{xcorr}\left\{\left|Z_{P_{o} P}\left(y_{w}\right)\right|,\left|Z_{P_{o} P}\left(y_{w}\right)\right|\right\}$,

$M=\max \left\{R_{y_{w}}\right\}$,

$L_{Z}=$ length $\left\{Z_{P_{o} P}\right\}$

$=$ length $\left.\left\{Z_{p q} \mid P_{o} \leq p \leq P ; 0 \leq q \leq p ; \bmod (p-|q|, 2)=0\right\}\right\}$

$\{p\}=\left\{P_{o}, \cdots, P\right\}=$ range of Zernike moments under test
$\left.\left|Z_{P_{o} P}\left(y_{w}\right)\right|=\left\{\left|Z_{p q}\left(y_{w}\right)\right| \mid P_{o} \leq p \leq P ; 0 \leq q \leq p ; \bmod (p-|q|, 2)=0\right\}\right\}$

$\left.\left|Z_{P_{o} P}\left(x_{w}\right)\right|=\left\{\left|Z_{p q}\left(x_{w}\right)\right| \mid P_{o} \leq p \leq P ; 0 \leq q \leq p ; \bmod (p-|q|, 2)=0\right\}\right\}$

noting that xcorr computes correlation of vectors (with equal length $=\mathrm{N}=L_{z}$ ) giving a vector of $2 L_{z}-1$ length without normalization as follows:

$R_{x y}(m)=\left\{\begin{array}{cc}\sum_{n=0}^{N-m-1} x_{n+m} y_{n} & m \geq 0 \\ R_{y x}(-m) & m<0\end{array}\right\}$

where $\mathrm{w}$ ranges over corresponding blocks of reference image (which represents the test image) and different poses of each person which are stored in the face database which represent the training set.

The Correlative Zernike Measure is defined as:

$E_{z}(y)=\arg \left[\max _{x}\left\{M_{y}\left[1-e_{c}(x, y)\right]\right\}\right]$

where

$M_{y}=\max \left[\max _{x}\left\{e_{c}(x, y)\right\}, \max _{x}\left\{e_{m}(x, y)\right\}\right.$

and the function $\arg [$.$] indicates the (ordinal number of the)$ person in the database whose some specific pose gives maximal similarity with the reference image $\mathbf{y}$. The above constant $M_{y}$ is defined jointly with the Min-Max Zernike error function $e_{m}(x, y)$ as explained below.

\section{B. Min-Max Zernike Measure}

Applying the following formulas to find Zernike error function between the corresponding blocks:

$e_{m}(x, y)=\min _{w}\left[\left\{M_{x_{w}}-M_{y_{w}}\right\}^{2}+\left\{\mu_{x_{w}}-\mu_{y_{w}}\right\}^{2}\right]$

where:

$$
\begin{aligned}
& M_{x_{w}}=\max \left|Z_{P_{o} P}\left(x_{w}\right)\right| \\
& \mu_{x_{w}}=\min \left|Z_{P_{o} P}\left(x_{w}\right)\right|
\end{aligned}
$$

We can define the Min-Max Zernike Measure as follows: $E_{m}(y)=\arg \left[\max _{x}\left\{M_{y}-e_{m}(x, y)\right\}\right]$

\section{Structural Similarity Measure}

As we mentioned above, the SSIM measurement can be applied to corresponding blocks of reference image and different poses giving the similarity function:

$S(x, y)=\max _{w} \operatorname{SSIM}\left(x_{w}, y_{w}\right)$

from which the Structural Similarity Measure is defined as follows:

$E_{s}(y)=\arg \left[\max _{x}\left\{M_{y} \cdot S(x, y)\right\}\right]$

\section{Combined Similarity Measures}

Based on the above three basic measures [Equations (9), (12), and (14)], we derive four Combined Similarity Measures as follows:

$$
\begin{aligned}
& e_{o}(y)=\arg \left[\max _{x}\left\{E_{1}(x, y) \cdot E_{2}(x, y) \cdot S(x, y) / M_{y}\right\}\right. \\
& e_{h}(y)=\arg \left[\max _{x}\left\{E_{2}(x, y) \cdot S(x, y)\right]\right. \\
& e_{n}(y)=\arg \left[\max _{x}\left\{E_{1}(x, y) \cdot S(x, y)\right]\right. \\
& e_{v}(y)=\arg \left[\max _{x}\left\{E_{1}(x, y) \cdot E_{2}(x, y) / M_{y}\right\}\right] \\
& \text { noting that: } \\
& E_{1}(x, y)=M_{y}-e_{c}(x, y)
\end{aligned}
$$




$$
E_{2}(x, y)=M_{y}-e_{m}(x, y)
$$

where the function $\arg [$.$] indicates the ordinal number of$ the person in the database as stated before, hence, it is the recognition function.

\section{E. Classification and Probability of Recognition}

Many techniques may be used for classification stage such as K-means or Naïve Bayesian, which is considered as a probabilistic approach. In this work, a new threshold is derived based on using a set of seven measures as defined in Equations (9), (12), (14)-(18). A success $(\mathrm{D}=1$ in our algorithms, which is the recognition of the face image as belonging to the data set) is reached when at least two measures recognize the reference face image from $\mathrm{C}=14$ cases [seven measurements for local analysis with seven measurements for semi-global analysis]. So, the threshold of belonging (recognition) is $T=2 / C$.

Now we define our confidence in this recognition and call it Probability of Recognition (belonging), $P_{r}$. First, we find the second peak (maximum) in the above measures. Then we find the difference between the absolute maximum and the second maximum for each measure, which we call here the MM difference. Normalization for these differences (by maximum difference) is necessary. The resulting quantity is the MMdifference for that measure. For example, $d_{z}(y)$ is the MMdifference for the Zernike Correlation Measure; $d_{m}(y)$ is the MM-difference for the Min-Max measure; and so on. Then, the probability of recognition is defined as follows:

$$
\begin{aligned}
& P_{r}(y) \\
& =\frac{\text { length }\left\{d_{i}(y) \mid i=\text { successful measure with } d_{i}(y)>\frac{1}{C}\right\}}{C}
\end{aligned}
$$

where "successful" means passing the Threshold $T=2 / C$. Hence, $P_{r}$ calculates how much confidence we should put in this recognition, noting that $\frac{1}{C}$ means at least one measure recognizes the image.

\section{F. Database Sets}

1) The AT\&T: This face image database contains 10 different images (poses) of each person; the set consist of images for 40 persons taken at different illuminations, rotation and facial expressions and facial details like glasses. The size of each image is $92 \times 112$ pixels, with 256 grey levels per pixel.

2) FEI Face Database: The FEI face database is a Brazilian face database that contains a set of face images taken between June 2005 and March 2006 at the Artificial Intelligence Laboratory of FEI in Sao Bernardo do Campo, Sao Paulo, Brazil. There are 14 images for each of 200 individuals, a total of 2800 images. All images are colorful and taken against a white homogenous background in an upright frontal position with profile rotation of up to about 180 degrees. Scale might vary about $10 \%$ and the original size of each image is $640 \times 480$ pixels. All faces are mainly represented by students and staff at FEI, between 19 and 40 years old with distinct appearance, hairstyle, and adorns. The number of male and female subjects are exactly the same and equal to 100 [22]. See Fig(2).

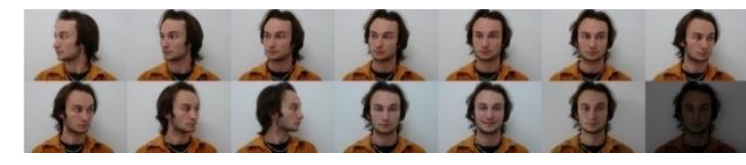

Fig. 2. Various face poses for a single person from The FEI Face Database.

\section{RESULTS AND DISCUSSION}

The performance of the proposed methodology is compared with two different benchmark datasets as well as using images taking at unconditional environment (may be hard environments if we consider the high degree of rotation and complex emotions). The results illustrate the efficacy of Zernike moments for the face recognition problems. The algorithm has been tested using approaches:

A. Under verification branch where the test image represents person belong to the training dataset, then the program must be to back all poses related with that person. At this testing the recognition rate reach to $98.9 \%$ (see Table 1) with ORL database, but with FEI Face Database (Brazilian) degrade to $96.5 \%$ (see Table 2).

B. Under classification branch where the tested image may be belong or not belong to the data set, the recognition rate may be reach to $95 \%$ based on the complexities of image.

A Comparison: The following table illustrate a comparison among a number of existing face recognition algorithms under similar databases.

Proposed Algorithm vs. Existing Algorithms.

\begin{tabular}{|l|l|l|l|}
\hline \multicolumn{1}{|c|}{ Paper } & \multicolumn{1}{c|}{ Algorithm(s) } & Database & $\%$ Recog \\
\hline $\begin{array}{l}\text { Guang Dai et al. } \\
(2003)[23]\end{array}$ & $\begin{array}{l}\text { Support Vector } \\
\text { Machines }\end{array}$ & AT\&T & 94.5 \\
\hline $\begin{array}{l}\text { A. Nabatchian et al. } \\
(2008)[24]\end{array}$ & $\begin{array}{l}\text { pseudo Zernike } \\
\text { moments }\end{array}$ & AT\&T & $95 \%$ \\
\hline $\begin{array}{l}\text { N. Farajzadeh et al. } \\
(2010)[25]\end{array}$ & Zernike moments & FERET & $94.3 \%$ \\
\hline $\begin{array}{l}\text { Zhan Shi et al. } \\
(2012)[26]\end{array}$ & $\begin{array}{l}\text { Pseudo-Zernike } \\
\text { Moment }\end{array}$ & AT\&T & $89 \%$ \\
\hline $\begin{array}{l}\text { Sara Nazari et al. } \\
(2013)[27]\end{array}$ & $\begin{array}{l}\text { Global and Local } \\
\text { Gabor Features }\end{array}$ & AT\&T & $91.8 \%$ \\
\hline $\begin{array}{l}\text { Raman Kumar et al. } \\
(2013)[28]\end{array}$ & PCA & Indian & $90 \%$ \\
\hline Proposed Algorithm & Zerinke and SSIM & AT\&T & $98.75 \%$, \\
\hline Proposed Algorithm & Zerinke and SSIM & Brazilian & $96.57 \%$ \\
\hline
\end{tabular}

\section{Discussion:}

When the query subject is in an unconstrained imaging environment, the true accept rate can fall from $99 \%$ to below $60 \%$ [29], so we can claim that our method attains higher accuracy than other method. Of course, it's difficult to determine the accurate rate, since the environment is un conditional (see Fig.3, where we used non-standard, self-made face images).

Experiments show that, in the image plane, when rotation exceeds $4^{\circ}$, the recognition rate drops rapidly, and the face image will be beyond recognition when rotation is more than $12^{\circ}$ [30]. However, using the proposed algorithm, face recognition attains high degrees of probability in spite of such rotations, where we might reach $99 \%$. 


\section{CONCLUSIONS}

1) Zernike moments started with order $p=$ zero give acceptable recognition rate that reaches $94.5 \%$. Low orders of Zernike moments are useful for face expression recognition.

2) Zernike moments started with $p=t w o$ give excellent recognition rate that reaches to $99 \%$.

3) Any Zernike moments beyond five returrn the same results (this means that the best range for Zernike moment is order [2-5].

4) Pure SSIM gives poor results, while modified SSIM, which combined edge detection methods with SSIM, gives better results.

5) Some of the above measurements are considered as weak measurements when they are used alone, but when combining these measurements with others excellent results are obtained.

6) Last conclusion is that: image blurring or noise does not affect these measures. In addition, they are very resistant against rotation.

\section{REFERENCES}

[1] Arnold Wiliem, Vamsi Krishna Madasu, Wageeh Boles \& Prasad Yarlagadda, "A face recognition approach using Zernike Moments for video surveillance", Queensland University of Technology website, http://eprints.qut.edu.au, 2007.

[2] W. Zhao, R. Chellappa, and P. J. Phillips, "Face Recognition: A Literature Survey", ACM Computing Surveys, Vol. 35, No. 4, pp. 399-458, 2003.

[3] Kresimir Delac, Mislav Grgic, and Sonja Grgic, "A comparative study of PCA, ICA AND LDA," International Journal of Imaging Systems and Technology, 2005.

[4] Zhengjun Pan and Hamid Bolouri, "High Speed Face Recognition Based on Discrete Cosine Transform and Neural Networks", University of Hertfordshire, UK, 1999.

[5] Ziad M. Hafid and Martin D. Levine, "Face Recognition Using the Discrete Cosine Transform", International Journal of Computer Vision 43(3), Kluwer Academic Publishers, 2001.

[6] Hazim Ekenel and Rainer Stiefelhagen, "Local Appearance Based Face Recognition Using Discrete Cosine Transform", 13th European Signal Processing Conference (EUSIPCO), 2005.

[7] Osslan Osiris Vergara Villegas, Mitzel Avíles Vianey Guadalupe Cruz Sánchez, and Humberto de, "A Novel Evolutionary Face Recognition Algorithm Using Particle Swarm Optimization", Fifth International Conference on Signal Image Technology and Internet Based Systems, 2009.

[8] Lanzarini Laura, La Battaglia Juan, Maulini Juan, and Hasperué Waldo, " Face Recognition Using SIFT and Binary PSO Descriptors", Proceedings of the ITI 2010 32nd Int. Conf. on Information Technology Interfaces, June 21-24, Cavtat, Croatia, 2010.

[9] Abhishek Sharma, Anamika Dubey, A. N. Jagannatha, R. S. Anand, "Pose invariant face recognition based on hybrid-global linear regression", Springer -Verlag London Limited, 2010.

[10] Qiang Zhang and Baoxin Li, "Discriminative K-SVD for Dictionary Learning in Face Recognition", IEEE Conference on Computer Vision and Pattern Recognition (CVPR), 2010.
[11] Manzoor Ahmad Lone, S. M. Zakariya, and Rashid Ali, "Automatic Face Recognition System by Combining Four Individual Algorithms", International Conference on Computational Intelligence and Communication Systems, 2011.

[12] Zhan Shi, Guixiong Liu, and Minghui Du, " Rotary Face Recognition Based on Pseudo-Zernike Moment", ECICE 2012, AISC 146, pp. 641646, Springer-Verlag Berlin Heidelberg, 2012.

[13] Sukhvinder Singh, Meenakshi Sharma, N Suresh Rao, "Accurate Face Recognition Using PCA and LDA", IJMIE, Volume 2, Issue 4, 2012.

[14] Manchula A., Arumugam S., "Robust Facial Data Recognition using multimodal Fusion Features in Multi-Variant Face Acquisition", International Journal of Computer Applications, Volume 64, No.11, 2013

[15] Chee-Way Chonga, P. Raveendranb, R.Mukundan, "Translation invariants of Zernike moments", Pattern Recognition 36, 2003.

[16] Sun-Kyoo Hwang, Whoi-Yul Kim, " Anovel approach to the fast computation of Zernike moments", Pattern Recognition 39, 2006.

[17] Z. Wang, A. C. Bovik, H. R. Sheikh, and E. P. Simoncelli, "Image quality assessment: From error visibility to structural similarity". IEEE Trans. Image Proc., 13: 600-612. DOI: 10.1109/TIP.2003.819861, Simoncelli, 2004.

[18] Asaad Noori Hashim and Zahir M. Hussain, " Novel Image Dependent Quality Assessment Measures", Journal of Computer Science 10 (9): 1548-1560, 2014.

[19] Seyed Mehdi Lajevardi, Zahir M. Hussain, "Automatic facial expression recognition: feature extraction and selection," Signal, Image and Video Processing, v.6, no.1, pp.159-169, 2012.

[20] Seyed Mehdi Lajevardi, Zahir M. Hussain, "Novel Higher-order Local Autocorrelation-like Feature Extraction Methodology for Facial Expression Recognition,” IET Image Processing, vol. 4, no. 2, 2010.

[21] Seyed Mehdi Lajevardi, Zahir M. Hussain, "Facial Expression Recognition Using Log-GaborFilters and Local Binary Pattern Operators," International Conference on Communication, Computer and Power (ICCCP'09), Muscat, Oman, 15-18 Feb. 2009.

[22] Personal Homepage of Dr. Carlos Eduardo Thomaz (Caru), http://fei.edu.br/ cet/facedatabase.html.

[23] Guang Dai and Changle Zhou, "Face Recognition Using Support Vector Machines with the Robust Feature", Proceedings of the 2003 IEEE International Workshop on Robot and Human interactive Communication Millbrae. Calimia, USA. Oct. 31 -Nov. 2,2003.

[24] A. Nabatchian, E. Abdel-Raheem and M. Ahmadi, "Human Face Recognition Using Different Moment Invariants: A Comparative Study", Congress on Image and Signal Processing. IEEE 2008.

[25] N. Farajzadeh, K. Faez, and G. Pan, "Study on the performance of moments as invariant descriptors for practical face recognition systems", IET Comput. Vis., 2010.

[26] Zhan Shi , Guixiong Liu and Minghui Du, "Rotary Face Recognition Based on Pseudo-Zernike Moment", Springer-Verlag Berlin Heidelberg 2012.

[27] Sara Nazari and Mohammad-Shahram Moin, "Face Recognition Using Global and Local Gabor Features", IEEE 2013.

[28] Raman Kumar and Satnam Singh, "Face Recognition using Principle Component Analysis for Biometric Security System", International Journal of Engineering Trends and Technology (IJETT) - Volume 4 Issue 9- Sep 2013

[29] Joshua C. Klontz Anil K. Jain, "A Case Study on Unconstrained Facial Recognition Using the Boston Marathon Bombings Suspects", Columbia Southern University, Computer Society, November, 2013.

[30] Shan, S.-G., Gao, W., "Curse of Miss-Alignment Problem in Face Recognition", Chinese Journal of Computers 05, 2005. 
TABLE I. Results of Appling Proposed Algorithm to ORL Data Set.

\begin{tabular}{|c|c|c|c|c|c|c|c|}
\hline \multirow{2}{*}{$\begin{array}{l}\text { perso } \\
\text { n }\end{array}$} & \multicolumn{2}{|c|}{ Successful poses } & \multicolumn{2}{|c|}{ Failed poses } & \multicolumn{2}{|c|}{ \% Success } & \multirow[t]{2}{*}{ Notes } \\
\hline & $\begin{array}{l}\text { Zernike } \\
\text { Moment } \\
\text { Ord 0-3 }\end{array}$ & $\begin{array}{l}\text { Zernike } \\
\text { Moment } \\
\text { Ord 2-5 }\end{array}$ & $\begin{array}{l}\text { Zernik } \\
\text { e } \\
\text { Mome } \\
\text { nt } \\
\text { Ord 0- } \\
3\end{array}$ & $\begin{array}{l}\text { Zernik } \\
\mathrm{e} \\
\text { Mome } \\
\mathrm{nt} \\
\text { Ord 2- } \\
5\end{array}$ & $\begin{array}{l}\text { Zernik } \\
\text { e } \\
\text { Mome } \\
\text { nt } \\
\text { Ord 0- } \\
3\end{array}$ & $\begin{array}{l}\text { Zernik } \\
\text { e } \\
\text { Mome } \\
\text { nt } \\
\text { Ord 2- } \\
5\end{array}$ & \\
\hline P1 & $10 / 10$ & $10 / 10$ & - & - & $100 \%$ & $100 \%$ & \multirow{10}{*}{$\begin{array}{l}\text { 1-Main problem } \\
\text { with persons } 4 \text { and } 10 .\end{array}$} \\
\hline P2 & $10 / 10$ & $10 / 10$ & - & - & $100 \%$ & $100 \%$ & \\
\hline P3 & $09 / 10$ & $10 / 10$ & 6 & - & $90 \%$ & $100 \%$ & \\
\hline P4 & $05 / 10$ & $10 / 10$ & $\begin{array}{l}1,3,4,7 \\
, 8\end{array}$ & - & $50 \%$ & $100 \%$ & \\
\hline P5 & $10 / 10$ & $09 / 10$ & - & 9 & $100 \%$ & $90 \%$ & \\
\hline P6 & $09 / 10$ & $10 / 10$ & 2 & - & $90 \%$ & $100 \%$ & \\
\hline P7 & $10 / 10$ & $10 / 10$ & - & - & $100 \%$ & $100 \%$ & \\
\hline P8 & $10 / 10$ & $10 / 10$ & - & - & $100 \%$ & $100 \%$ & \\
\hline P9 & $10 / 10$ & $9 / 10$ & - & 1 & $100 \%$ & $90 \%$ & \\
\hline P10 & $07 / 10$ & $10 / 10$ & $4,5,10$ & - & $70 \%$ & $100 \%$ & \\
\hline P11 & $10 / 10$ & $10 / 10$ & - & - & $100 \%$ & $100 \%$ & \multirow{10}{*}{$\begin{array}{l}\text { 1-Main problem } \\
\text { with person } \\
12 \text { and } 16 . \\
\text { 2-percentage of } \\
\text { success is 92\%. } \\
\text { With Zernike orders } \\
\text { from } \mathbf{0 - 3}\end{array}$} \\
\hline $\mathrm{P} 12$ & $06 / 10$ & $10 / 10$ & $1,4,7,8$ & - & $60 \%$ & $100 \%$ & \\
\hline P13 & $10 / 10$ & $10 / 10$ & - & - & $100 \%$ & $100 \%$ & \\
\hline P14 & $10 / 10$ & $10 / 10$ & - & - & $100 \%$ & $100 \%$ & \\
\hline P15 & $10 / 10$ & $10 / 10$ & - & - & $100 \%$ & $100 \%$ & \\
\hline P16 & $07 / 10$ & $10 / 10$ & $3,8,10$ & - & $70 \%$ & $100 \%$ & \\
\hline P17 & $09 / 10$ & $10 / 10$ & 5 & - & $90 \%$ & $100 \%$ & \\
\hline P18 & $10 / 10$ & $10 / 10$ & - & - & $100 \%$ & $100 \%$ & \\
\hline P19 & $10 / 10$ & $10 / 10$ & - & - & $100 \%$ & $100 \%$ & \\
\hline P20 & $10 / 10$ & $10 / 10$ & - & - & $100 \%$ & $100 \%$ & \\
\hline P21 & $10 / 10$ & 09/10 & - & 2 & $100 \%$ & $90 \%$ & \multirow{10}{*}{$\begin{array}{l}\text { 1-Ten poses of ten } \\
\text { persons are succeed } \\
\text { 2-percentage of } \\
\text { succeed is } 100 \% \\
\text { With Zernike orders } \\
\text { from } \mathbf{0 - 3}\end{array}$} \\
\hline P22 & $10 / 10$ & $10 / 10$ & - & - & $100 \%$ & $100 \%$ & \\
\hline $\mathrm{P} 23$ & $10 / 10$ & $10 / 10$ & - & - & $100 \%$ & $100 \%$ & \\
\hline P24 & $10 / 10$ & $10 / 10$ & - & - & $100 \%$ & $100 \%$ & \\
\hline $\mathrm{P} 25$ & $10 / 10$ & $9 / 10$ & - & 8 & $100 \%$ & $90 \%$ & \\
\hline P26 & $10 / 10$ & $10 / 10$ & - & - & $100 \%$ & $100 \%$ & \\
\hline P27 & $10 / 10$ & $10 / 10$ & - & - & $100 \%$ & $100 \%$ & \\
\hline $\mathrm{P} 28$ & $10 / 10$ & $10 / 10$ & - & - & $100 \%$ & $100 \%$ & \\
\hline P29 & $10 / 10$ & $10 / 10$ & - & - & $100 \%$ & $100 \%$ & \\
\hline $\mathrm{P} 30$ & $10 / 10$ & $10 / 10$ & - & - & $100 \%$ & $100 \%$ & \\
\hline P31 & $10 / 10$ & $10 / 10$ & - & - & $100 \%$ & $100 \%$ & \multirow{10}{*}{$\begin{array}{l}\text { 1-Main problem } \\
\text { with person } 40 \\
\text { 2-percentage of } \\
\text { succeed is } 96 \% \\
\text { With Zernike orders } \\
\text { from } \mathbf{0 - 3}\end{array}$} \\
\hline P32 & $10 / 10$ & $10 / 10$ & - & - & $100 \%$ & $100 \%$ & \\
\hline $\mathrm{P} 33$ & $10 / 10$ & $10 / 10$ & - & - & $100 \%$ & $100 \%$ & \\
\hline P34 & $10 / 10$ & $10 / 10$ & - & - & $100 \%$ & $100 \%$ & \\
\hline P35 & $09 / 10$ & $09 / 10$ & 1 & 2 & $90 \%$ & $90 \%$ & \\
\hline P36 & $10 / 10$ & $10 / 10$ & - & - & $100 \%$ & $100 \%$ & \\
\hline P37 & $10 / 10$ & $10 / 10$ & - & - & $100 \%$ & $100 \%$ & \\
\hline P38 & $10 / 10$ & $10 / 10$ & - & - & $100 \%$ & $100 \%$ & \\
\hline P39 & $10 / 10$ & $10 / 10$ & - & - & $100 \%$ & $100 \%$ & \\
\hline P40 & $07 / 10$ & $10 / 10$ & $1,6,10$ & - & $70 \%$ & $100 \%$ & \\
\hline Total & $388 / 400$ & $400 / 400$ & $22 / 400$ & $5 / 400$ & $94.5 \%$ & $\begin{array}{l}98.75 \\
\%\end{array}$ & \\
\hline
\end{tabular}


TABLE II. RESUlts of APPLing Proposed Algorithm to BraziLIAN DATA SET.

\begin{tabular}{|c|c|c|c|c|c|c|c|}
\hline Person & $\begin{array}{l}\text { Successful } \\
\text { Poses }\end{array}$ & $\begin{array}{l}\text { Failed } \\
\text { Poses }\end{array}$ & $\begin{array}{c}\text { Succeed } \\
\%\end{array}$ & Person & $\begin{array}{c}\text { Successfu } \\
1 \\
\text { Poses } \\
\end{array}$ & $\begin{array}{l}\text { Failed } \\
\text { Poses }\end{array}$ & $\begin{array}{c}\text { Succeed } \\
\%\end{array}$ \\
\hline $\mathrm{P} 1$ & $14 / 14$ & - & $100 \%$ & P26 & $12 / 14$ & 11,14 & $86 \%$ \\
\hline $\mathrm{P} 2$ & $14 / 14$ & - & $100 \%$ & $\mathrm{P} 27$ & $14 / 14$ & - & $100 \%$ \\
\hline P3 & $14 / 14$ & - & $100 \%$ & P28 & $14 / 14$ & - & $100 \%$ \\
\hline $\mathrm{P} 4$ & $13 / 14$ & 2 & $93 \%$ & P29 & $14 / 14$ & - & $100 \%$ \\
\hline P5 & $13 / 14$ & 10 & $93 \%$ & P30 & $14 / 14$ & - & $100 \%$ \\
\hline P6 & $13 / 14$ & 5 & $93 \%$ & P31 & $13 / 14$ & 10 & $93 \%$ \\
\hline P7 & $13 / 14$ & 6 & $93 \%$ & P32 & $13 / 14$ & 11 & $93 \%$ \\
\hline P8 & $14 / 14$ & - & $100 \%$ & P33 & $13 / 14$ & 12 & $93 \%$ \\
\hline P9 & $14 / 14$ & - & $100 \%$ & P34 & $14 / 14$ & - & $100 \%$ \\
\hline P10 & $14 / 14$ & - & $100 \%$ & $\mathrm{P} 35$ & $13 / 14$ & 13 & $93 \%$ \\
\hline P11 & $13 / 14$ & 8 & $93 \%$ & P36 & $13 / 14$ & 14 & $93 \%$ \\
\hline P12 & $14 / 14$ & - & $100 \%$ & P37 & $14 / 14$ & - & $100 \%$ \\
\hline P13 & $14 / 14$ & - & $100 \%$ & P38 & $14 / 14$ & - & $100 \%$ \\
\hline P14 & $14 / 14$ & - & $100 \%$ & P39 & $14 / 14$ & - & $100 \%$ \\
\hline P15 & $14 / 14$ & - & $100 \%$ & $\mathrm{P} 40$ & $14 / 14$ & - & $100 \%$ \\
\hline P16 & $13 / 14$ & 11 & $93 \%$ & P41 & $14 / 14$ & - & $100 \%$ \\
\hline P17 & $13 / 14$ & 10 & $93 \%$ & $\mathrm{P} 42$ & $13 / 14$ & 2 & $93 \%$ \\
\hline P18 & $13 / 14$ & 12 & $93 \%$ & P43 & $14 / 14$ & - & $100 \%$ \\
\hline P19 & $14 / 14$ & - & $100 \%$ & $\mathrm{P} 44$ & $12 / 14$ & 9,10 & $86 \%$ \\
\hline $\mathrm{P} 20$ & $13 / 14$ & 4 & $93 \%$ & $\mathrm{P} 45$ & $13 / 14$ & 10 & $93 \%$ \\
\hline $\mathrm{P} 21$ & $14 / 14$ & - & $100 \%$ & $\mathrm{P} 46$ & $14 / 14$ & - & $100 \%$ \\
\hline P22 & $13 / 14$ & 14 & $93 \%$ & P47 & $13 / 14$ & 4 & $93 \%$ \\
\hline $\mathrm{P} 23$ & $14 / 14$ & - & $100 \%$ & $\mathrm{P} 48$ & $14 / 14$ & - & $100 \%$ \\
\hline $\mathrm{P} 24$ & $14 / 14$ & - & $100 \%$ & P49 & $13 / 14$ & 6 & $93 \%$ \\
\hline P25 & $13 / 14$ & 12 & $93 \%$ & P50 & $14 / 14$ & - & $100 \%$ \\
\hline & & & & Total & $676 / 700$ & $24 / 700$ & $\% 96.57$ \\
\hline
\end{tabular}



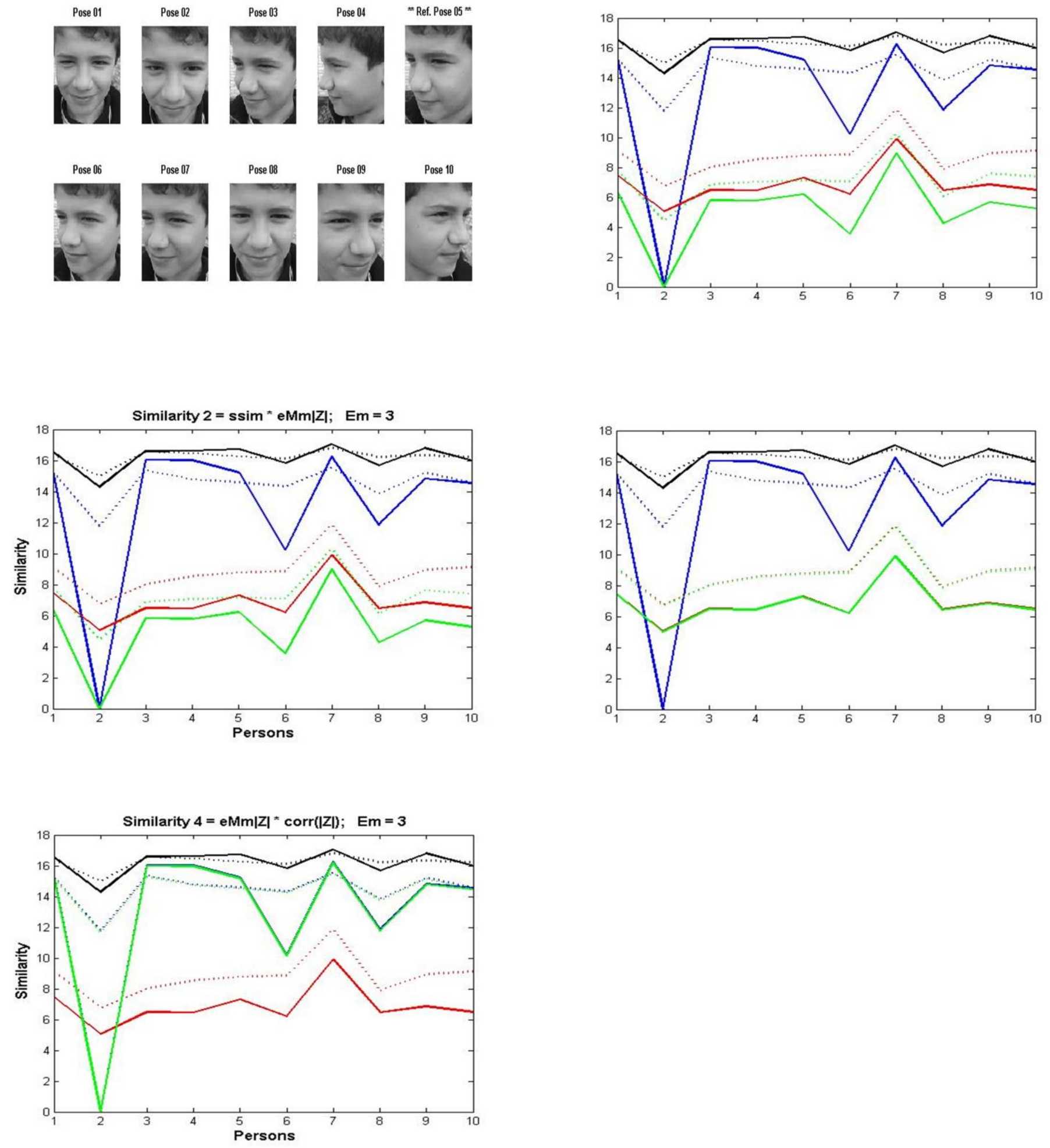

Fig. 3. Results with non-standard poses $(\mathrm{D}=1, \mathrm{Pe}=7, \mathrm{Pr}=0.5714)$. 

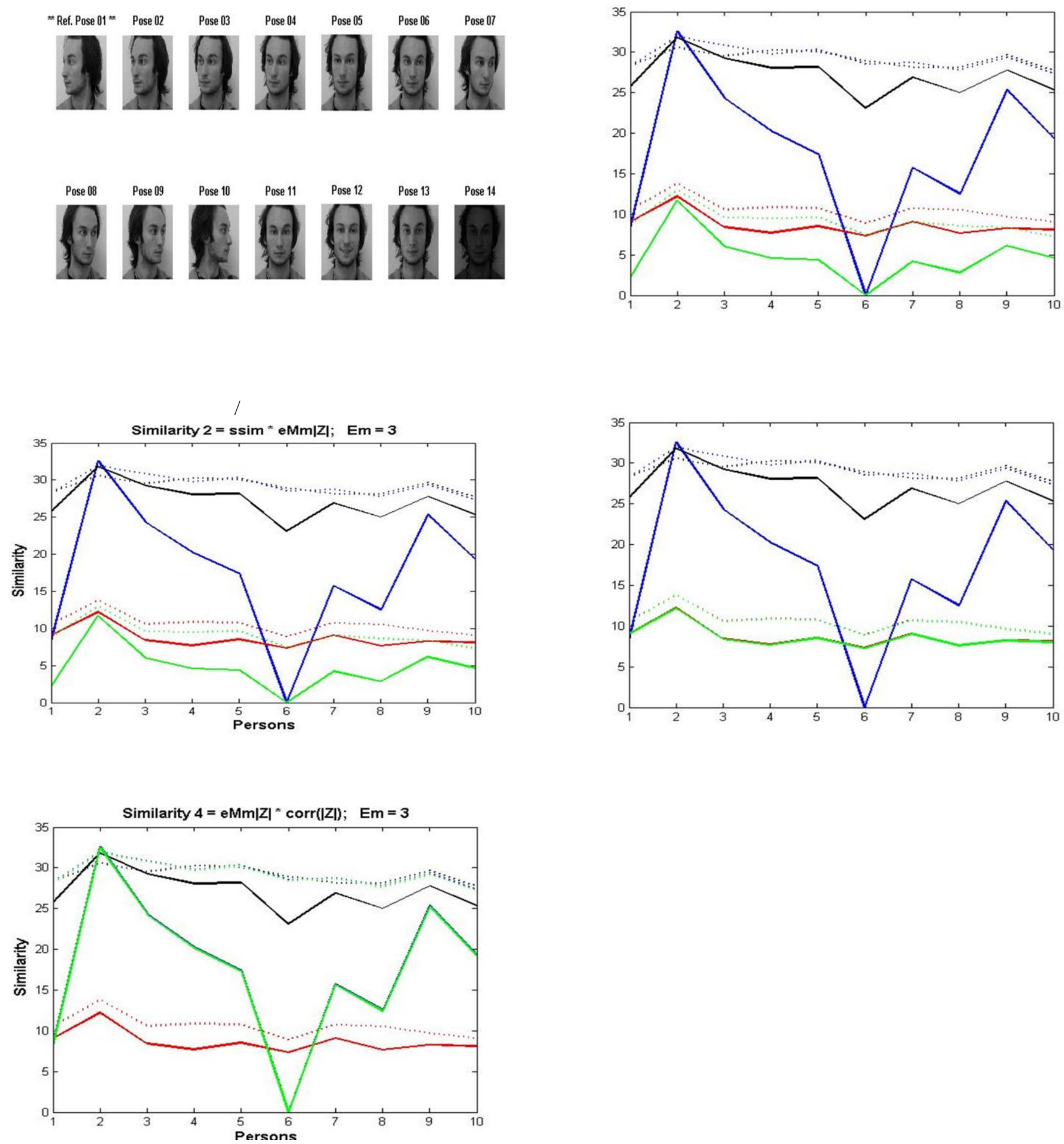

Fig. 4. Results with FEI Face Database(Brazilian) $(D=1, P e=2, P r=0.785)$ 

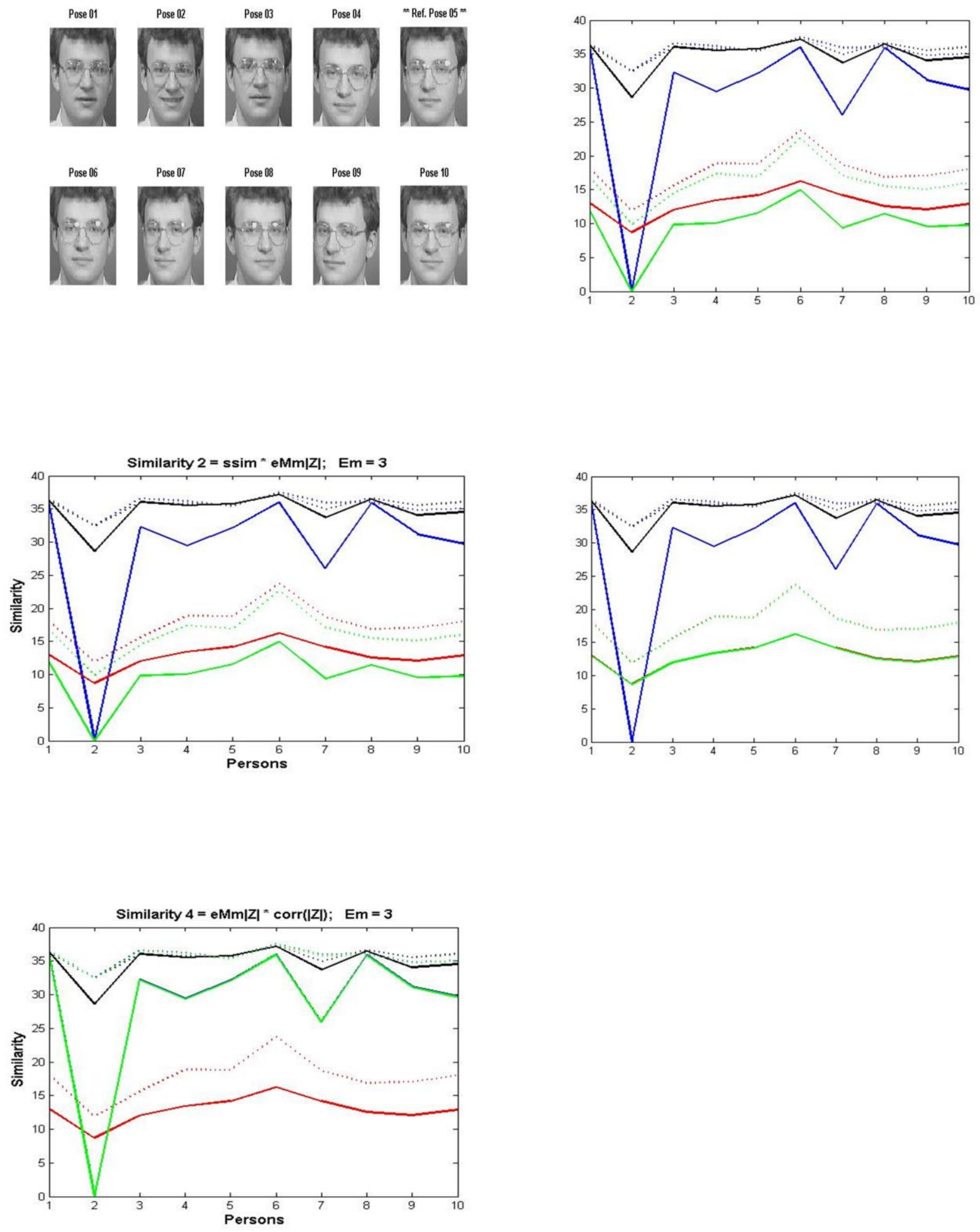

Fig. 5. Results with ORL Database $(\mathrm{D}=1, \mathrm{Pe}=6, \mathrm{Pr}=0.5714)$ 